\title{
Intervenções educativas para prevenção de acidentes em pré-escolares: revisão integrativa
}

Suelen Brito Azevedo ${ }^{1}$ Célia Maria Ribeiro de Vasconcelos², Luciana Pedrosa Leal ${ }^{3}$, Maria Gorete Lucena de Vasconcelos ${ }^{4}$

\section{RESUMO}

Os acidentes mais prevalentes em pré-escolares são atropelamentos, afogamentos, quedas, ferimentos e queimaduras. Cuidadores, professores e profissionais da saúde envolvidos no cuidado a essas crianças devem estar centrados em ações de prevenção. O objetivo deste estudo foi investigar as intervenções educativas para prevenção de acidentes em pré-escolares descritas na literatura científica. Revisão integrativa realizada nas bases de dados Scopus, Web of Science, CINAHL, Medline, LILACS, IBECS, BDENF, CUIDEN e na biblioteca virtual Cochrane, utilizando os descritores educação em saúde, prevenção de acidentes e pré-escolares. Foram selecionados 19 estudos, sendo que 13 destes incluíram cuidadores como público-alvo. As tecnologias educacionais mais empregadas foram os jogos, vídeos, materiais informativos, aconselhamentos e palestras. Evidenciou-se que, a adequada seleção da abordagem pedagógica e da tecnologia educacional considerando o contexto sociodemográfico e cultural do público-alvo pode promover melhor efeito no conhecimento nas intervenções educativas acerca da prevenção de acidentes.

Descritores: Educação em Saúde; Educação Infantil; Pré-Escolar; Prevenção de Acidentes; Enfermagem Pediátrica.

\footnotetext{
${ }^{1}$ Enfermeira, Mestre em Enfermagem. Discente do Programa de Pós-Graduação em Enfermagem, nível Doutorado, da Universidade Federal de Pernambuco. Recife, PE, Brasil. E-mail: suelenbritoazevedo@gmail.com.

2 Enfermeira, Mestre em Saúde Coletiva. Discente do Programa de Pós-Graduação em Enfermagem, nível Doutorado, da Universidade Federal de Pernambuco. Professor Ensino Básico Técnico Tecnológico do Instituto Federal de Educação, Ciência e Tecnologia de Pernambuco - Campus Pesqueira. Pesqueira, PE, Brasil. E-mail: cmrvasconcelos@gmail.com.

${ }^{3}$ Enfermeira, Doutora em Nutrição. Professora Adjunta da Universidade Federal de Pernambuco. Recife, PE, Brasil. E-mail: lucianapleal@hotmail.com.

${ }^{4}$ Enfermeira, Doutora em Enfermagem em Saúde Pública. Professora Associada da Universidade Federal de Pernambuco. Recife, PE, Brasil. E-mail: mariagoretevasconcelos@gmail.com.
}

Artigo recebido: 25/07/2018.

Artigo aprovado: 15/08/2018.

Artigo publicado: 31/12/2018.

\section{Como citar esse artigo:}

Azevedo SB, Vasconcelos CMR, Leal LP, Vasconcelos MGL. Intervenções educativas para prevenção de acidentes em pré-escolares: revisão integrativa. Rev. Eletr. Enf. [Internet]. 2018 [acesso em: ];20:v20a56. Disponível em: https://doi.org/10.5216/ree.v20.47978. 


\section{INTRODUÇÃO}

A Organização Pan-Americana de Saúde (OPAS) ${ }^{(1)}$ recomenda que durante o período da infância deve-se garantir o crescimento e desenvolvimento saudáveis das crianças promovendo a saúde e reduzindo as morbidades e incapacidades, nos quais se enquadram os acidentes não intencionais. Os acidentes são eventos não intencionais e evitáveis causadores de lesões físicas e/ou emocionais oriundos do ambiente doméstico ou social(2).

“No mundo, mais de um milhão de crianças morrem por ano devido a acidentes não intencionais. No Brasil, os mesmos representam a principal causa de morte de crianças e adolescentes menores de 14 anos com 3,8 mil mortes e 116 mil crianças hospitalizadas por ano ${ }^{(3-4)}$." Os acidentes podem ser previsíveis e passíveis de prevenção primária, secundária e terciária mediante ações para contribuir na diminuição da incidência, prevalência e da gravidade dos danos ocasionados por este agravo à criança(5-6).

No Brasil, dentre as principais causas de acidentes na infância estão os acidentes de trânsito, afogamentos, sufocações, quedas, queimaduras e intoxicações. No ano de 2014, o acidente de trânsito foi a principal causa de morte entre crianças e adolescentes (um a 14 anos) abrangendo $39 \%$ dos $\operatorname{casos}^{(7)}$. Segundo o Departamento de Informática do Sistema Único de Saúde (DATASUS) no mesmo ano, quanto aos dados de morbidade por acidentes entre crianças e adolescentes (um a 14 anos), foram 49\% por quedas, 17\% queimaduras, $11 \%$ acidentes de trânsito, $3 \%$ intoxicação e $20 \%$ por outras causas (traumas, afogamentos, etc.) ${ }^{(8)}$.

Os acidentes mais prevalentes nos pré-escolares (dois a seis anos de idade), principalmente na faixa etária de dois a cinco anos, são acidentes de trânsito, quedas, queimaduras e outros acidentes (ferimento, acidentes com animais, traumas $)^{(9)}$. Neste contexto, os pais, familiares, profissionais da educação e da saúde envolvidos nos diversos espaços de convívio da criança como domicílio, escola, parque, creche/pré-escola/centro ou núcleo de educação infantil, entre outros, devem estar atentos e adotar ações de prevenção dos acidentes reconhecendo os diversos fatores de risco e de proteção(6).

No Brasil, os Ministérios da Saúde e da Educação, por meio do Programa Saúde na Escola (PSE), vinculam os profissionais da atenção primária em saúde e da educação para o desenvolvimento de estratégias de promoção da saúde, incluindo a prevenção de doenças e agravos e atenção à saúde para o enfrentamento das vulnerabilidades, acidentes e/ou violências, que comprometem o desenvolvimento das crianças ${ }^{(10)}$.

Dentre os profissionais da atenção primária, o enfermeiro exerce um papel essencial na mudança de comportamentos e hábitos saudáveis junto à comunidade, através de ações educativas sobre os meios de prevenção de doenças e agravos ${ }^{(11)}$. A adoção de intervenções educativas com as famílias e comunidade para a promoção da segurança nos espaços sociais poderá favorecer a redução dos riscos para acidentes ${ }^{(8)}$.

A promoção de intervenções educativas sobre a prevenção de acidentes em pré-escolares é oportuna, uma vez que nesta faixa etária, que corresponde dos 24 aos 59 meses de idade ${ }^{(9)}$, as crianças ampliam suas habilidades sociais e motoras, explorando mais os ambientes, movidas por curiosidade, incapacidade de prevenir situações de perigo devido ao desenvolvimento cognitivo e motor em formação, bem como limitações na noção corporal e espacial e falta de coordenação motora fina ${ }^{(12)}$.

Nesta perspectiva, o objetivo deste estudo foi investigar as intervenções educativas para prevenção de acidentes em pré-escolares descritas na literatura científica. 


\section{MÉTODO}

Revisão integrativa elaborada seguindo seis etapas distintas: (1) Identificação do problema/formulação da pergunta norteadora do estudo; (2) Amostragem/busca na literatura; (3) Categorização dos estudos/coleta de dados; (4) Análise dos dados; (5) Discussão/interpretação dos resultados e (6) Apresentação da revisão integrativa $^{(13)}$.

Utilizou-se a seguinte questão de pesquisa: Quais as intervenções educativas para prevenção de acidentes em pré-escolares descritas em artigos científicos disponíveis no meio eletrônico?

A busca foi realizada em junho de 2018 por dois pesquisadores separadamente utilizando os Descritores em Ciências da Saúde (DeCS) e suas traduções padronizadas pelo Medical Subject Heading (MeSH), por meio dos unitermos isolados e combinados com o operador booleano "and" nas línguas portuguesa, inglesa e espanhola: "educação em saúde/health education/educación em salud", "prevenção de acidentes/accident prevention/prevención de accidentes" e "pré-escolar/child preschool/preescolar".

As bases de dados pesquisadas foram: Medical Literature Analysis and Retrieval Sistem on-line (Medline); Elsevier API (Scopus); Web of Science; Cumulative Index of Nursing and Allied Health Literature (CINAHL); Literatura Latino-Americana e do Caribe em Ciências da Saúde (LILACS); Base de dados em Enfermagem (BDENF); Índice Bibliográfico Espanhol de Ciências de Saúde (IBECS); Fundación Index (CUIDEN) e a Biblioteca Cochrane Library.

Adotaram-se como critérios de inclusão: estudos originais, publicados nos idiomas português, inglês e espanhol, sem estabelecer o limite de tempo de publicação. Os critérios de exclusão foram: resumos de pesquisa, relato de casos, editoriais, artigos de jornais, revisão de literatura, reflexões teóricas, teses e dissertações. 0 cruzamento dos descritores educação em saúde, prevenção de acidentes e pré-escolares nas bases de dados resultou em 1.010 estudos.

Para seleção da amostra foram lidos os títulos e/ou resumos dos 1.010 estudos e selecionados 55 que estavam em consonância com a pergunta norteadora. Destes, 11 estudos estavam em duplicata em duas ou mais bases de dados, totalizando 44 estudos. Dentre os estudos pré-selecionados, 20 foram excluídos, cinco por serem artigos de revisão ou discussão, um livro, um editorial e 13 em outros idiomas, resultando numa amostra de 24 estudos para leitura na íntegra e obtenção dos dados (Figura 1).

Na coleta de dados foi utilizado o instrumento proposto por Ursi, que contempla: título, periódico (ano, volume, número e páginas), autores, tipo de publicação, características metodológicas, objetivos, amostra, tratamento dos dados, resultados, conclusões ${ }^{(14)}$. Os estudos foram avaliados quanto ao rigor metodológico através de um instrumento adaptado do Critical Appraisal Skills Programme (CASP) com 10 itens pontuáveis quanto aos objetivos do estudo, clareza na descrição dos métodos empregados, definição dos participantes, critérios de inclusão/exclusão, cumprimento dos aspectos éticos, coleta de dados, processo de análise, resultados e limitações ${ }^{(15)}$. Este instrumento categoriza os estudos em: nível A (6 a 10 pontos) - boa qualidade metodológica e viés reduzido; nível B (até 5 pontos) - qualidade metodológica adequada, porém com risco de viés aumentado. Foram excluídos cinco dos 24 estudos, uma vez que foram classificados no nível B, obtendo-se uma amostra final de 19 estudos. 
Figura 1: Fluxograma da amostragem e seleção dos estudos da revisão integrativa.

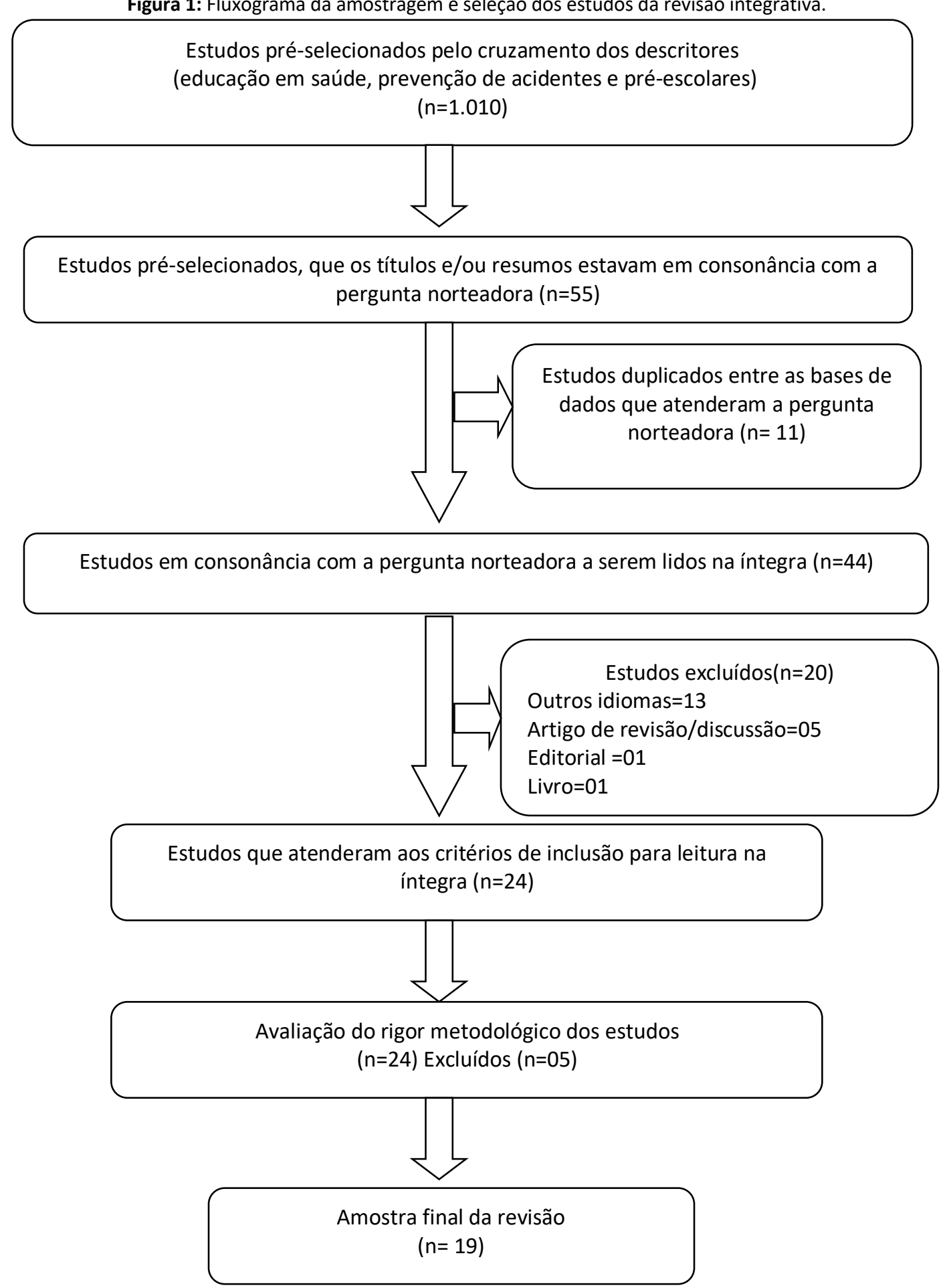

Quanto ao nível de evidência os estudos foram analisados pela classificação hierárquica segundo a abordagem metodológica adotada, distribuídos em sete níveis:

- $\quad$ Nível I (revisões sistemáticas ou metanálises);

- Nível Il (os ensaios clínicos randomizados controlados);

- Nível III (os ensaios clínicos sem randomização);

- Nível IV (estudos de coorte ou caso-controle);

- Nível V (revisões sistemáticas de estudos descritivos e qualitativos);

- Nível VI (estudos descritivos ou qualitativos); 
- $\quad$ Nível VII (opiniões de autoridades e/ou relatórios de comitês de especialidades)(16).

Os estudos foram agrupados segundo o público-alvo selecionado para intervenção educativa sobre prevenção de acidentes-cuidadores; cuidadores e pré-escolares; e pré-escolares. O termo "cuidadores" compreende os pais ou responsáveis e familiares da criança. Para análise e discussão dos resultados foi utilizada a literatura científica acerca das abordagens pedagógicas em intervenções educacionais(17-18).

\section{RESULTADOS}

Dos 19 estudos, 12 se enquadraram no nível de evidência $\|^{(19-30)}$, cinco no nível III(31-35) e dois no nível VI(3637). A maioria das intervenções educativas foi direcionada para os cuidadores ${ }^{(19-25,31-33)}$, seguidos dos préescolares $^{(28-30,34-35,37)}$, cuidadores e pré-escolares ${ }^{(26-27,36)}$. Os locais selecionados para a execução das intervenções educativas foram escolas(26-31,34-35,37), $\operatorname{hospitais}^{(19,21,23,32)}$, $\operatorname{domicílios}^{(25,33)}$, $\operatorname{comunidades}^{(20,22,28,36)}$ e domicílio/comunidade(24).

As tecnologias evidenciadas nos estudos foram vídeos, materiais informativos, carta, pôsteres, ilustrações, computadores interativos, jogos de simulação(19,21-23,25-30,32-34,36-37). Desses estudos, dois(33,36) que utilizaram materiais informativos com palestras e $u^{(26)}$, a aplicação de um vídeo educativo para as famílias, não apresentaram desfechos satisfatórios entre os cuidadores.

O Quadro 1 sintetiza aspectos dos estudos selecionados e incluídos na revisão descrevendo informações sobre autor/ano, base de dados, objetivos, método/recurso utilizado/local do estudo e principais resultados. Os artigos também foram separados de acordo com o público-alvo envolvido nas intervenções educativas. 


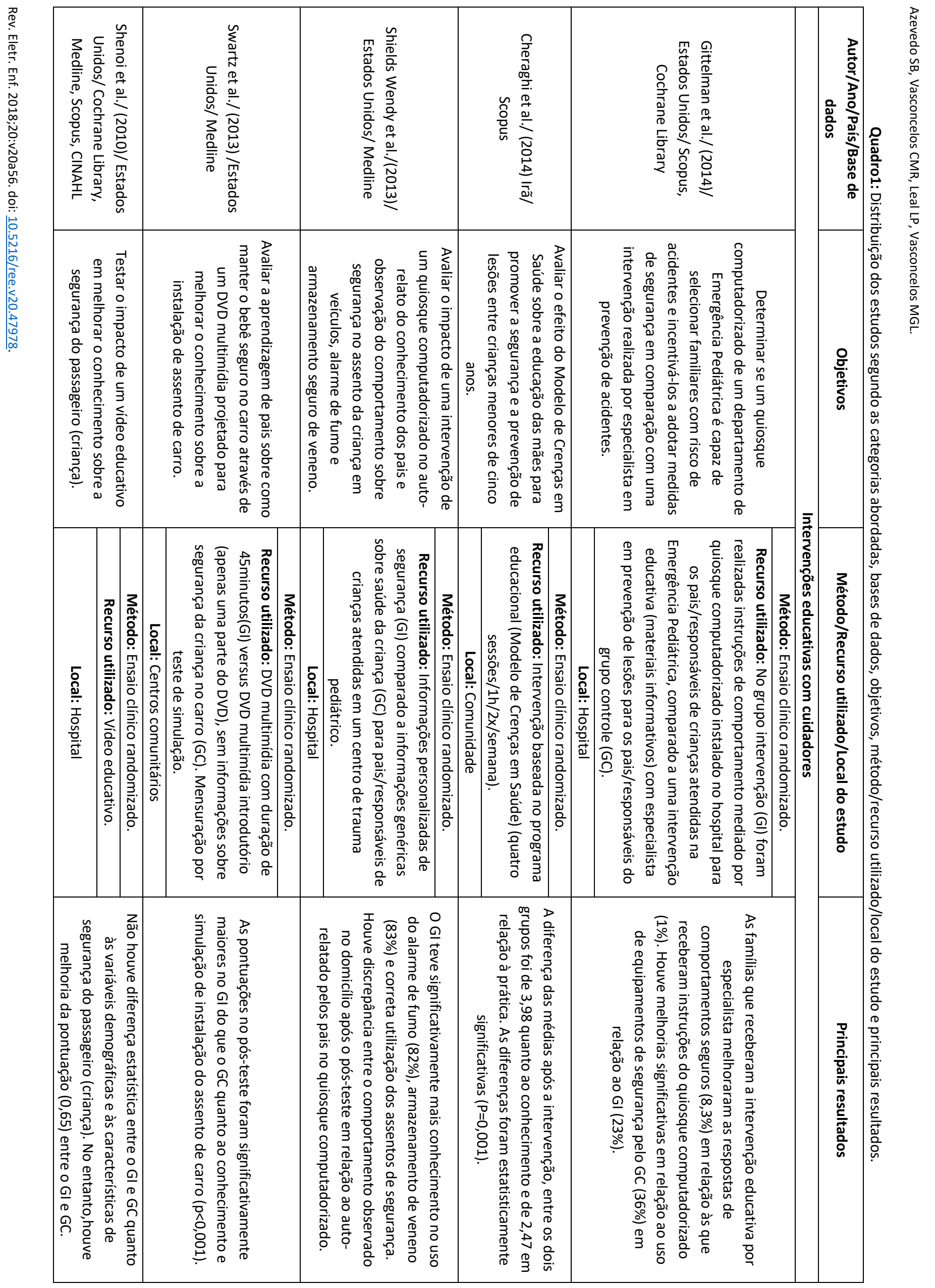




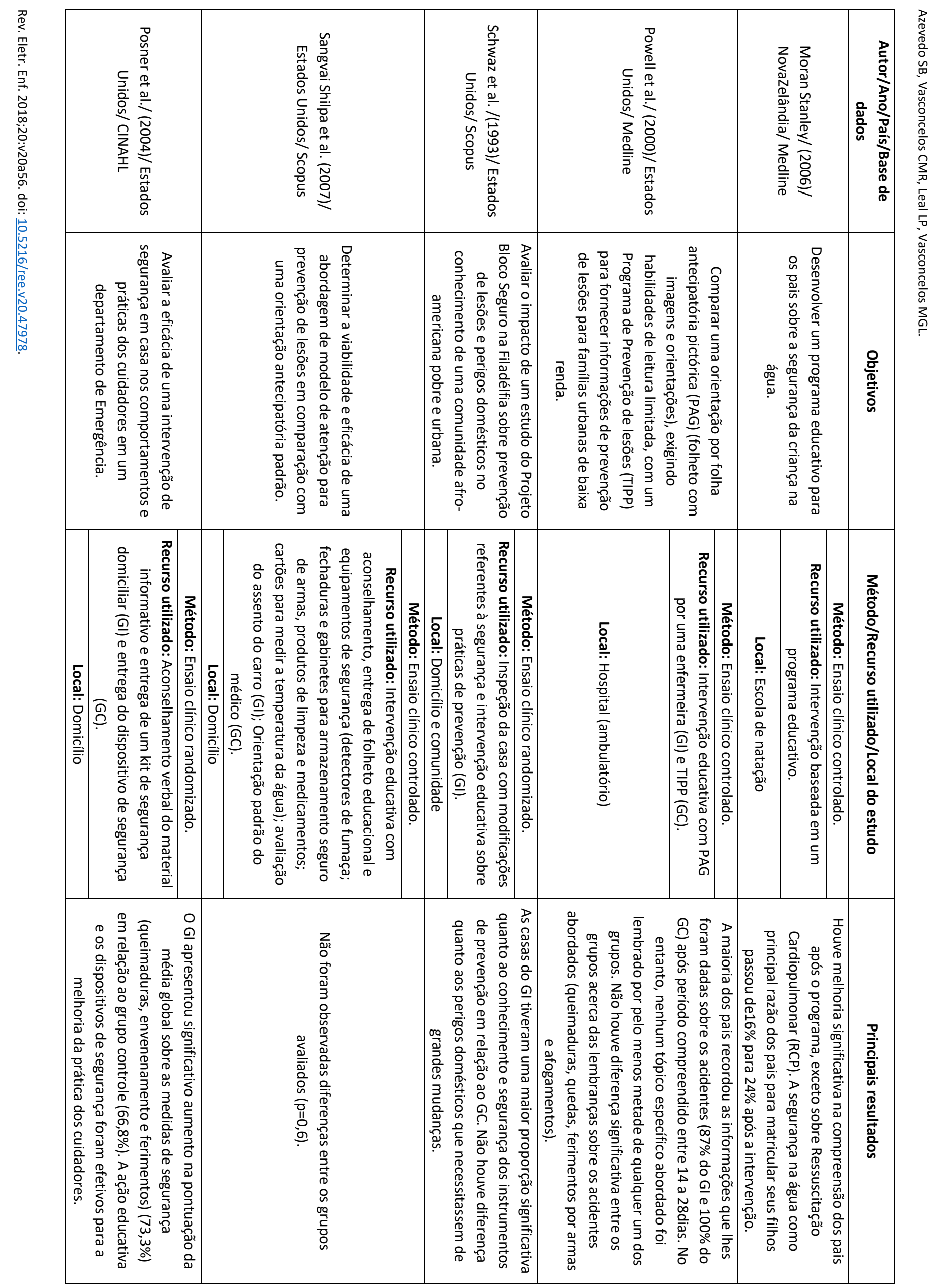




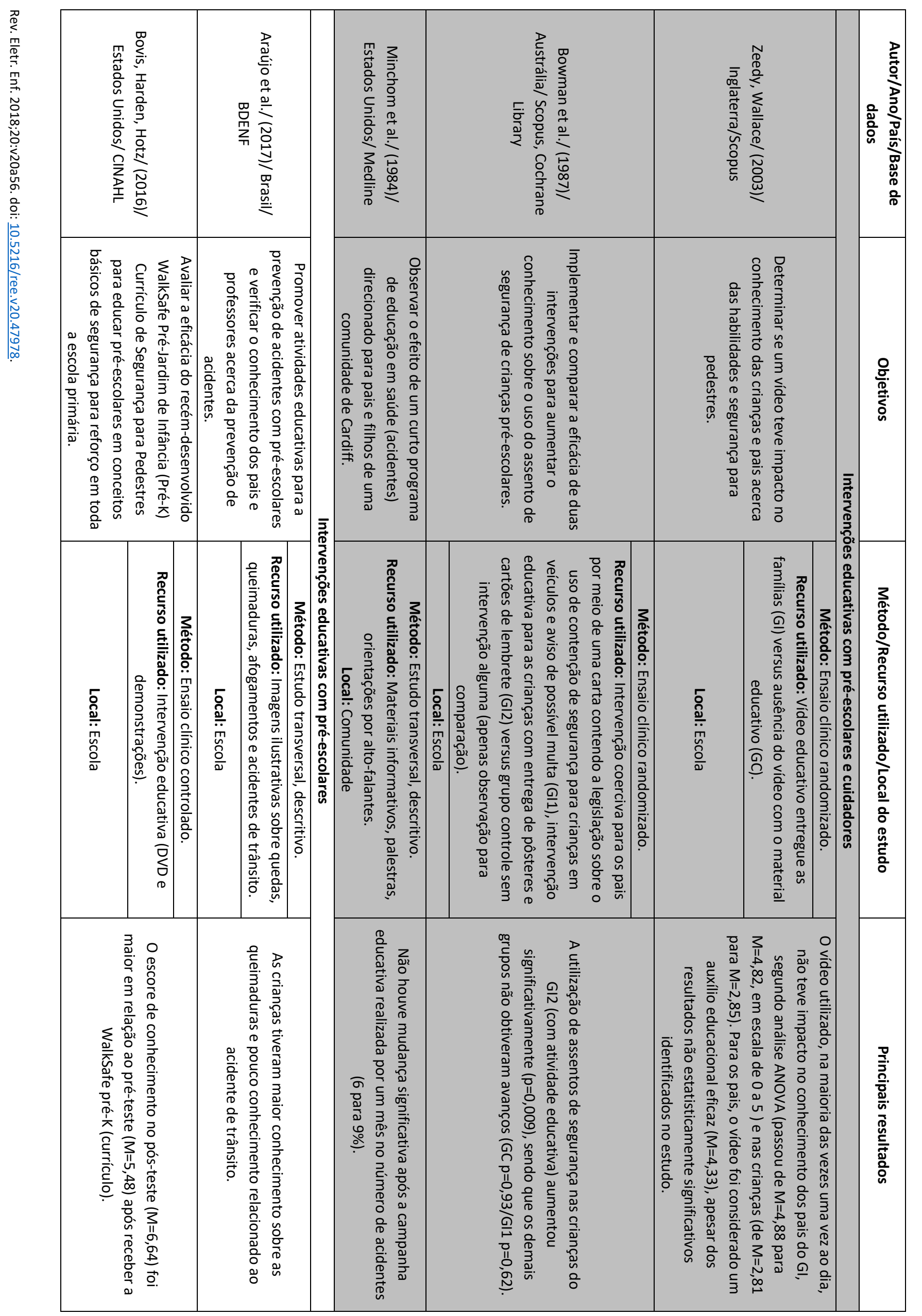




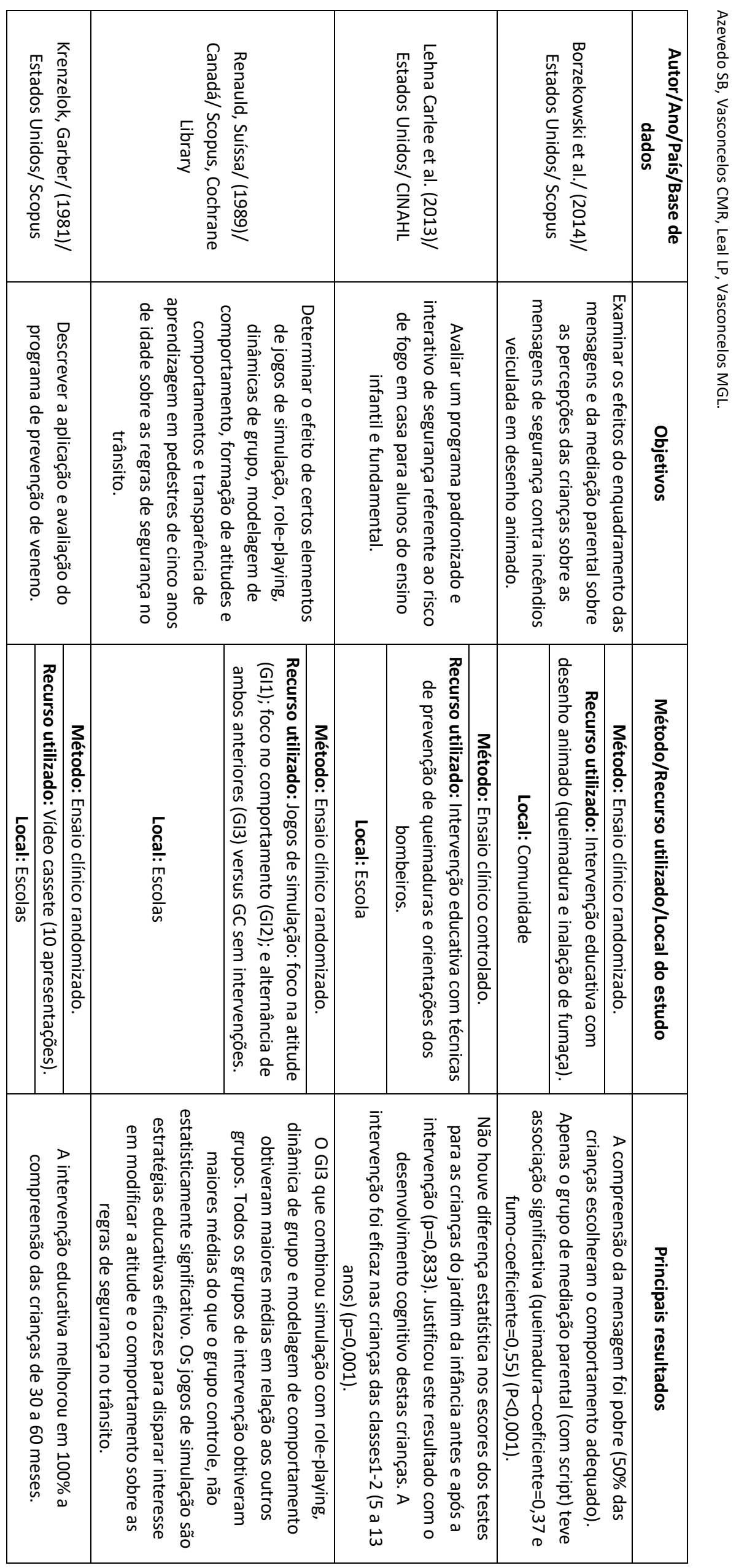




\section{DISCUSSÃO}

As intervenções desenvolvidas com maior frequência com fins de educação em saúde para prevenção de acidentes foram programas educacionais ${ }^{(19-21,31-32,35-36)}$, uso de materiais informativos impressos (folhetos, pôsteres, cartões) $)^{(25,27,33,36-37)}$ e de jogos ou vídeos/DVD (22-23,26,28-30,34). Entretanto, nem todos os estudos evidenciaram resultados positivos ${ }^{(26,33,36)}$. Essas intervenções educativas ${ }^{(19-37)}$ foram analisadas quanto à construção do conhecimento dos cuidadores e pré-escolares apoiada nas abordagens pedagógicas, que podem ser mediadas por tecnologias educacionais para melhoria efetiva da aprendizagem ${ }^{(38)}$.

Os atores da rede social da criança constituem o público-alvo das intervenções educativas promovidas por profissionais da saúde, em virtude de frequentemente estes agravos acontecerem/durante momentos de desatenção dos pais ou responsáveis e familiares associados ou não ao comportamento de risco dos préescolares ${ }^{(39)}$. Intervenções educativas mediadas por profissionais como palestras ${ }^{(21,24,35)} \mathrm{e}$ associadas ao uso de tecnologias ${ }^{(19,25,27,32-33,36)}$ são capazes de melhorar o conhecimento e habilidades dos cuidadores na prevenção de acidentes. Por outro lado, o uso das tecnologias educacionais sem o envolvimento do facilitador e/ou em campanhas veiculadas por meio da distribuição de materiais informativos não levam em consideração as necessidades individuais e o contexto de cada família, o que pode repercutir em menor efeito no conhecimento dos cuidadores e pré-escolares ${ }^{(26,36)}$.

Na construção de novas tecnologias educacionais utilizadas nas abordagens pedagógicas, o conteúdo deve ser adequadamente avaliado, de fácil compreensão, levando em consideração os contextos sociais, demográficos, econômicos e culturais do público-alvo ${ }^{(40)}$. Essa relevância se evidencia no melhor efeito do conhecimento ao se utilizar um DVD multimídia quando comparado ao uso da mesma ferramenta com informações básicas ${ }^{(22)}$.Assim como na ocorrência de desfechos positivos ou negativos, quando não se leva em conta, por exemplo, a renda e o nível de escolaridade da população(32,41).

Por outro lado, intervenções educativas com abordagem pedagógica tradicional, baseada na transferência de conteúdos, sem considerar a participação dos educandos na produção do conhecimento, apesar de mediada por tecnologia educacional, podem ocasionar desmotivação entre os educandos ${ }^{(17)}$. Ensinar não é apenas transferir conhecimentos, mas criar possibilidades para a própria produção ou construção(17), como evidenciado na promoção de uma intervenção educativa sobre a segurança no trânsito (pôsteres e cartões de lembrete) para as crianças, que aumentou significativamente o aprendizado quando comparadas a uma intervenção coerciva (carta com aviso de multa iminente) junto aos pais, que não aderiram às medidas de segurança da criança no transporte por carro(27).

A abordagem educacional focada no educando é mais efetiva na construção do conhecimento e mudanças de comportamentos dos cuidadores ${ }^{(21,25)}$,e escolares ${ }^{(37)}$ sobre a prevenção de acidentes. Em atividades educacionais voltadas às crianças é fundamental despertar a sua atenção, por meio de metodologias lúdicas ${ }^{(42)}$, para a mudança de comportamento. Essa atitude estimula a compreensão de como agir para garantir sua segurança em ambientes que ofereçam risco para acidentes como no carro, sobretudo frente ao uso do assento $^{(27)}$.

As crianças em idade pré-escolar nesta fase de desenvolvimento cognitivo, denominada de préoperacional, caracterizada pelo pensamento simbólico, podem não ter um aprendizado significativo diante da 
abordagem pedagógica e do recurso tecnológico na intervenção educativa ${ }^{(18,26,27,36)}$. A utilização de metodologias ativas de ensino permeadas pelas atividades lúdicas como jogos, brincadeiras de faz de conta, uso do brinquedo, pinturas e desenhos para os pré-escolares tornam-se medidas eficazes para estimular as ações preventivas e, consequentemente, diminuir os acidentes na infância(28-29,37,43).

Segundo Piaget, no estágio pré-operacional do desenvolvimento cognitivo em crianças de dois a 6/7 anos de idade, predominam a intuição, impressão e percepção através do pensamento simbólico, que podem ser desenvolvidos nos desenhos, brincadeiras de faz de conta e por imitação ${ }^{(44)}$. Nesta fase, a abordagem educacional permeada pelo lúdico permite que a aprendizagem seja eficiente e efetiva, porque desperta, intencionalmente, a imaginação do pré-escolar sobre a compreensão da sua realidade, possibilitando-o ao fenômeno denominado de transcendência, no qual serão originados significados e conhecimentos acerca dos acidentes não-intencionais ${ }^{(45)}$. Para professores da educação infantil, orientar e educar as crianças é fundamental a utilização do lúdico na prevenção dos acidentes ${ }^{(41,45)}$, o que torna o processo de aprendizagem mais dinâmico facilitando a compreensão(29-30,37).

Todavia, outras atividades utilizadas na intervenção educativa não tradicional como entrega de kits, explicação e observação da prática, possibilitaram o conhecimento adequado dos pré-escolares sobre ouso do assento de segurança no veículo ${ }^{(27)}$.Esses resultados apontam que,independentemente da atividade proposta, esta deve estar alinhada às metodologias ativas de ensino-aprendizagem como estratégias viáveis para as intervenções de prevenção de acidentes.Os desenhos animados e vídeos educativos implementados na intervenção educativa, sem a mediação do educador, podem ser percebidos pela criança apenas como um entretenimento, não gerando impacto no processo de aprendizagem ${ }^{(26,28)}$. O desafio é o desenvolvimento de animações interativas, em que a criança possa se manifestar e que possibilite mudanças de comportamento mais seguras e em consonância às boas práticas em saúde.

O jogo educativo de simulação foi o recurso mais adequado para a fase do desenvolvimento cognitivo em crianças com cinco anos de idade, ao combinar role-playing, dinâmica de grupo e modelagem de comportamento para modificar a atitude e comportamento quanto as regras de segurança no trânsito ${ }^{(29)}$. As intervenções educativas sobre os acidentes não intencionais foram benéficas quando empregaram jogo interativo, jogos de simulação e vídeos educativos ${ }^{(29-30,34)}$.

Os profissionais de educação infantil assumem a responsabilidade por estas crianças durante as atividades desenvolvidas no ambiente escolar. A escolha do tipo de abordagem pedagógica e tecnologia educacional a ser utilizada na avaliação do conhecimento dos profissionais da educação infantil são essenciais para efetividade dos resultados e planejamento das ações relacionadas à prevenção de acidentes no ambiente escolar. Na compreensão de professores, as ações de prevenção de acidentes devem ser realizadas por meio da vigilância ativa (atenção e cuidado imediato) e mudanças no ambiente físico (mesas, cadeiras, piso, paredes, banheiros e móveis), promovidas no cotidiano das creches para oferecer segurança aos pré-escolares ${ }^{(41)}$.

A predominância das intervenções educativas para prevenção de acidentes no ambiente escolar (26-27,29-31,34${ }^{35,37)}$ deve-se ao fato dos pré-escolares passarem uma parte ou o dia todo do seu cotidiano (matutino ou vespertino) neste ambiente, favorecendo a sua aplicabilidade neste público-alvo e seus cuidadores ${ }^{(46)}$. Vale considerar a consonância entre o ambiente abordado (domicílio, escola, parque, rua, entre outros), as 
características do público-alvo e a abordagem educativa mais adequada, a fim de obter desfecho favorável na implementação de intervenções educativas promovendo a saúde e, consequentemente, a diminuição da

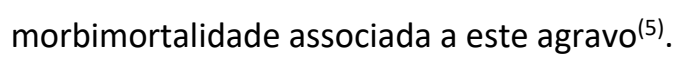

Nesta revisão, foi observada uma lacuna de estudos que envolvam em uma mesma pesquisa cuidadores formais, professores e outros profissionais participantes no cuidado à criança na educação infantil. Nesse sentido, novas pesquisas devem ser incentivadas envolvendo, não apenas os cuidadores a esse grupo específico de crianças, mas também as pessoas envolvidas na educação infantil. Os cuidadores formais, devido às configurações familiares em que a mãe está inserida no mercado de trabalho, são os responsáveis pelos cuidados diários à criança e devem ser capazes de prevenir os acidentes domésticos - quedas, queimaduras, intoxicação, engasgo, trauma, entre outros.

A escola, sendo o local mais utilizado para as intervenções educativas nesta revisão, pode ser considerada como um ambiente promotor da saúde, que possibilita ações de triagem e encaminhamento para os serviços de saúde, diagnóstico clínico ou social e a efetivação de ações interdisciplinares e intersetoriais na oferta de capacitações para os professores por profissionais da atenção primária ${ }^{(47)}$. A articulação da Estratégia Saúde da Família (ESF) ao PSE é fundamental para contribuir na formação integral das crianças e adolescentes através de estratégias de promoção da saúde, prevenção de doenças e agravos e enfrentamento das vulnerabilidades, acidentes e/ou violências, que possam comprometer o desenvolvimento das crianças $^{(10)}$.

A educação em saúde possibilita a construção do conhecimento, baseado na participação e no diálogo, com o objetivo de valorizar os saberes das pessoas, e não apenas o conhecimento científico, baseadas na reflexão e construção da consciência crítica sobre a sua própria saúde e da comunidade ${ }^{(48)}$. Nas intervenções permeadas pela educação em saúde se produzo conhecimento, que consiste em saber sobre determinado evento e na habilidade de aplicá-lo para a resolução de problemas. Essa ação é relevante por estimular que os indivíduos assumam atitudes, tenham modo de proceder, com opiniões, predisposições, e consequentemente, adotem práticas na aplicação de regras e princípios de uma ciência ou arte em determinada situação(49).

O enfermeiro e demais profissionais da saúde são corresponsáveis no desenvolvimento de ações de promoção à saúde da criança na escola e devem estar capacitados para utilizar as tecnologias no processo de ensino e aprendizagem. A evolução dos meios de comunicação permitiu o incremento de novas tecnologias, vídeos/DVD $^{(22-23,26,28,30,34)}$, computadores ${ }^{(19,21)}$ e smartphones, como ferramentas educacionais. A associação entre as palestras ou aconselhamentos com os materiais informativos, vídeos ou computadores facilita o desenvolvimento das intervenções educativas para os pré-escolares, cuidadores e profissionais da educação

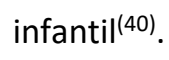

A preocupação no desenvolvimento de intervenções educativas para a segurança no trânsito de crianças enquanto passageiras (assentos no carro) ou pedestres evidenciada nos estudos ${ }^{(21-23,26-27,29,34)}$ está relacionada às altas taxas de incidência dos acidentes de trânsito entre crianças no mundo ${ }^{(3)}$.No entanto, a redução da taxa de mortalidade por este tipo de acidente no Brasil declinou da 10ạ para a 11a posição do ranking dos países no período de 1990 a 2015, envolvendo crianças menores de cinco anos. Este efeito é decorrente de várias ações preventivas como a melhoria da sinalização de trânsito nas vias, o uso obrigatório da cadeirinha a partir de 2008 , 
a fiscalização dos órgãos responsáveis e as campanhas educativas mediadas por organizações governamentais e não-governamentais ${ }^{(50)}$.

A proposição de políticas públicas específicas sobre a prevenção de acidentes na infância articuladas com as organizações não-governamentais existentes (Safe Kids, Criança Segura) é fundamental, com o intuito de planejar e implementar ações intersetoriais para conscientização das pessoas envolvidas no cuidado com as crianças nos diversos ambientes de convivência.Todos os estudos ${ }^{(19-37)}$ enfatizaram a importância das ações de prevenção primária para minimizar os fatores de risco, oferecer segurança nos diversos espaços de convivência das crianças (domicílio, escola, comunidade) e, consequentemente, para diminuir a incidência dos acidentes nãointencionais. Os fatores de risco podem estar associados à idade, sexo e falta de percepção do risco da criança antes dos sete anos de idade, ao conhecimento, atitude e prática dos cuidadores, à estrutura física e materiais adequados nos ambientes bem como a inexistência de ações de prevenção ${ }^{(39)}$.

Uma das limitações evidenciadas nesta revisão foi não permitir a ampliação da avaliação do efeito das tecnologias educacionais para a população em geral pelo fato da maioria dos estudos serem ensaios clínicos com públicos-alvo específicos. Outro aspecto a ser considerado refere à inexistência de registros da prática dessas tecnologias em instituições de ensino e/ou órgãos governamentais.

\section{CONCLUSÕES}

As intervenções educativas foram predominantemente direcionadas para os cuidadores dos pré-escolares, tornando-se evidente a necessidade de promover mais pesquisas com intervenções educativas sobre prevenção de acidentes para os cuidadores formais e profissionais da educação infantil, presentes no cotidiano, e integrantes da rede social dos pré-escolares. No que diz respeito ao uso das tecnologias educacionais, as mais utilizadas foram os programas instrutivos, materiais informativos e vídeos/DVD. Nas intervenções educativas aplicadas aos cuidadores, apenas a palestra ou a mesma associada à tecnologia educacional foi o método de ensino mais eficaz.

Outro aspecto a ser considerado é a abordagem pedagógica baseada nas metodologias ativas de ensino, que demonstraram maior efetividade das intervenções educacionais. Novas pesquisas devem ser executadas para verificar a adequabilidade na seleção de metodologias ativas associadas às tecnologias educacionais, principalmente em ensaios clínicos, a fim de comprovar a efetividade das intervenções educacionais contextualizadas ao público-alvo. O local mais comum para a realização dos estudos foi a escola, permitindo a prática de ações intersetoriais como o PSE, para favorecer a troca de saberes entre os profissionais da saúde (enfermeiros, médicos, odontólogos) e da educação. O enfermeiro como um dos profissionais da atenção primária ao atuar nas ações de educação em saúde poderá transformar a escola em um ambiente promotor da saúde dos pré-escolares e, assim, minimizar os fatores de risco e, consequentemente, a incidência dos acidentes na infância.

\section{REFERÊNCIAS}

1. Organização Panamericana de Saúde (OPAS). The Lancet Early Childhood Development. Advancing Early Childhood Development: from Science to Scale. An Executive Summary for The Lancet's Series [Internet]. Washington: OPAS, 2016 [acesso em: 20 abril 2018]. Disponível em: https://www.thelancet.com/pb-

assets/Lancet/stories/series/ecd/Lancet ECD Executive Summary.pdf. 
2. Ministério da Saúde. Sistema de Vigilância de Violências e Acidentes (Viva): 2009, 2010 e 2011. Secretaria de Vigilância em Saúde, Departamento de Vigilância de Doenças e Agravos não Transmissíveis e Promoção da Saúde. [Internet]. Brasília; DF, 2013 [acesso em: 10 ago. 2016]. Disponível em:http://bvsms.saude.gov.br/bvs/publicacoes/sistema vigilancia violencia acidentes.pdf. 3. CICEL. Grupo de Coordenação e Manutenção. Classificação Internacional de causas externas de lesões. Versão 1.2. Adelaide: Consumer Safety Institute, Amsterdam and AlHW National Injury Surveillance Unit. [Internet]. 2004 [acesso em: 15 jan. 2017]. Disponível em: http://www.rivm.nl/who-fic/ICECI/CICEL\%20port.pdf.

4. DATASUS - Departamento de Informática do SUS. [Internet]. Brasília: Ministério da Saúde. 2015 [acesso em: 20 abril 2018]. Disponível em: http://www2.datasus.gov.br/DATASUS/index.php?area=0205.

5. Filócomo FRF, Harada MJCS, Mantovani R, Ohara CVS. Perfil dos acidentes na infância e adolescência atendidos em um hospital público. Acta Paul Enferm. [Internet] 2017; 30(3): 287-94. [acesso em: 31 maio 2018]. Disponível:

http://www.scielo.br/pdf/ape/v30n3/1982-0194-ape-30-03-0287.pdf.

6. Gurgel AKC, Monteiro Al. Prevenção de acidentes domésticos infantis: susceptibilidade percebida pelas cuidadoras. Cuidado é fundamental. [Internet]. 2016; 8(4): 5126-5135. [acesso em: 18 agosto 2018]. Disponível em:

http://www.seer.unirio.br/index.php/cuidadofundamental/article/view/5021/pdf 1.

7. DATASUS - Tecnologia da Informação a serviço da saúde, Notas técnicas. Óbitos por causas externas - Brasil. [Internet]. Brasília: Ministério da Saúde. 2014 [acesso em:10 fev. 2016]. Disponível em:

http://tabnet.datasus.gov.br/cgi/deftohtm.exe?sim/cnv/obt10uf.def.

8. DATASUS - Tecnologia da Informação a serviço da saúde, Notas técnicas. Morbidade hospitalar do SUS. [Internet]. Brasília: Ministério da Saúde. 2014 [acesso em:10 fev. 2017]. Disponível em:

http://tabnet.datasus.gov.br/cgi/deftohtm.exe?sih/cnv/niuf.def.

9. Malta et al. A ocorrência de causas externas na infância em serviços de urgência: aspectos epidemiológicos, Brasil, 2014. Ciência \& Saúde Coletiva. [Internet]. 2016; 21(12): 3729-3744. [acesso em: 17 ago. 2018]. Disponível em:

https://www.scielosp.org/pdf/csc/2016.v21n12/3729-3744/pt.

10. Ministério da Saúde. Caderno do gestor do PSE. Ministério da Saúde, Ministério da Educação. [Internet]. 2015 [acesso em: 12 dez. 2016]. Disponível em: http://bvsms.saude.gov.br/bvs/publicacoes/caderno gestor pse.pdf.

11. Barbiani R, Dalla Nora CR, Schaefer R. Nursing practices in the primary health care context: a scoping review. Rev. Latino-Am. Enfermagem. [Internet]. 2016; 24: e2721. [acesso em: 21 abril 2018]. Disponível em: http://www.scielo.br/pdf/rlae/v24/pt 01041169-rlae-24-02721.pdf.

12. Malta et al. Atendimentos por acidentes e violências na infância em serviços de emergências públicas. Cad. Saúde Pública. [Internet]. 2015; 31 (5): 1095-1105. [acesso em: 28 jun. 2016]. Disponível em: http://www.scielo.br/pdf/csp/v31n5/0102-311X-csp31-5-1095.pdf.

13. Soares et al. Revisão integrativa: conceitos e métodos utilizados na Enfermagem. Rev Esc Enferm USP. [Internet]. 2014; 48(2): 335-45. [acesso em: 17 ago. 2018]. Disponível em: http://www.periodicos.usp.br/reeusp/article/view/84097/86950.

14. Ursi ES, Gavão CM. Prevenção de lesões de pele no perioperatório: revisão integrativa da literatura. Rev Latino-am Enfermagem [Internet]. 2006; 14(1): 124-31. [acesso em: 17 ago. 2018]. Disponível em: http://www.scielo.br/pdf/rlae/v14n1/v14n1a17.

15. Toledo MM. Vulnerabilidade de adolescentes ao HIV/AIDS: Revisão integrativa [dissertação]. São Paulo: Universidade de São Paulo, Escola de Enfermagem. 2008.

16. Stillwell S, Melnyk BM, Fineout-Overholt E, Williamson K. Evidence-Based Practice: Stepbystep. American Journal of Nursing. [Internet]. 2010; 110(5): 41-7. [acesso em: 17 jun. 2016]. Disponível em: http://europepmc.org/abstract/MED/20179464.

17. Freire P. Pedagogia da autonomia: saberes necessários à prática educativa. São Paulo: Paz e Terra; 2008.

18. Freire P, Shor I. Medo e ousadia: cotidiano do professor. Rio de Janeiro: Paz e terra; 1987. [acesso em: 02 ago. 2016]. Disponível em: http://www.pucrs.br/edipucrs/online/autonomia/autonomia/3.6.html.

19. Gittelman MA, Pomerantz WJ, McClanahan N, Damon A, Ho M. A computerized kiosk to teach injury prevention: Is it as effective as human interaction? Journal of trauma and acute care surgery. [Internet]. 2014; 77(3): S2-S7. [acesso em: 17 jun. 2016 ]. Disponível em:

http://journals.Iww.com/jtrauma/Abstract/2014/09001/A computerized kiosk to teach injury prevention .2.aspx.

20. Cheraghi P, Poorolajal J, Hazavehi SM, Rezapur-Shahkolai F. Effect of educating mothers on injury prevention among children aged $<5$ years using the Health Belief Model: a randomized controlled trial. Public Health. [Internet]. 2014; 128 (9): 825-30. [acesso em: 18 jan. 2017]. Disponívelem: http://www.publichealthjrnl.com/article/S0033-3506(14)00154-1/pdf.

21. Shields WC, McDonald EM, McKenzie L, Wang MC, Walker AR, Gielen AC. Utilizing the pediatric emergency department to deliver tailored safety messages: Results of a randomized controlled trial. Pediatric emergency care. [Internet]. $2013 ; 29$ (5): 628. [acesso em: 17 jun. 2016]. Disponível em: http://www.ncbi.nlm.nih.gov/pmc/articles/PMC3674582/pdf/nihms460139.pdf.

22. Swartz L, Glang A, Schwebel DC, Geiger Wolfe EG, Gau J, Schroeder S. Keeping baby safe: A randomized trial of a parent training program for infant and toddler motor vehicle injury prevention. Accident Analysis \& Prevention. [Internet].2013; 60: 35-41. [acesso em: 17 jun. 2016]. Disponível em: http://www.sciencedirect.com/science/article/pii/S0001457513002947. 
23. Shenoi R, Saz EU, Jones JL, Ma L, Yusuf S. An emergency department intervention to improve knowledge of child passenger safety. Pediatric emergency care. [Internet]. 2010; 26 (12): 881-887. [acesso em: 02 ago. 2016]. Disponível em:

http://journals.Iww.com/pec-online/Abstract/2010/12000/An Emergency Department Intervention to Improve.2.aspx.

24. Schwaz DF, Grisso JA, Miles C, Holmes JH, Sutton RL. An injury prevention program in an urban African-American community. American Journal of Public Health. [Internet]. 1993; 83 (5): 675-680. [acesso em: 02 ago. 2016]. Disponível em: http://ajph.aphapublications.org/doi/pdf/10.2105/AJPH.83.5.675.

25. Posner JC, Hawkins LA, Garcia-Espana F, Durbin DR. A randomized, clinical trial of a home safety intervention based in an emergency department setting. Pediatrics. [Internet]. 2004; 113 (6): 1603-1608. [acesso em: 02 ago. 2016]. Disponível em: http://pediatrics.aappublications.org/content/113/6/1603.short.

26. Zeedy KMS, Wallace L. Tackling children's road safety through edutainment: an evaluation of effectiveness. Health Education Research. [Internet]. 2003; 18 (4): 493-505. [acesso em: 02 ago. 2016]. Disponível em:

http://her.oxfordjournals.org/content/18/4/493.full.pdf+html.

27. Bowman JA, Sanson-Fisher RW, Webb GR. Interventions in preschools to increase the use of safety restraints by preschool children. Pediatrics. [Internet]. 1987; 79 (1): 103-109. [acesso em: 02 ago. 2016]. Disponível em:

http://pediatrics.aappublications.org/content/79/1/103.short.

28. Borzekowski D, Clearfield E, Rimal R, Gielen A. Young children's perceptions of fire-safety messages: do framing and parental mediation matter?J Burn Care Res. [Internet]. 2014; 35 (4): 303-12. [acesso em: 02 fev. 2017]. Disponível em: https://www.ncbi.nlm.nih.gov/pubmed/23877137.

29. Renauld L, Suíssa S. Evaluation of the efficacy of simulation games in traffic safety education of kindergarten children. American Journal of Public Health. [Internet]. 1989; 79 (3): 307-309. [acesso em: 02 ago. 2016]. Disponível em:

http://ajph.aphapublications.org/doi/pdf/10.2105/AJPH.79.3.307.

30. Krenzelok EP, Garber RJ. Teaching poison prevention to preschool children, their parents, and professional educators through child care centers. American journal of public health. [Internet]. 1981; 71(7): 750-752. [acesso em:02 ago. 2016]. Disponível em: http://ajph.aphapublications.org/doi/pdf/10.2105/AJPH.71.7.750.

31. Moran K, Stanley T. Toddler drowning prevention: teaching parents about water safety in conjunction with their child's in-water lessons. International journal of injury control and safety promotion. [Internet]. 2006; 13 (4): 254-256. [acesso em: 02 ago. 2016 ]. Disponível em: http://www.tandfonline.com/doi/pdf/10.1080/17457300600678201.

32. Powell EC, Tanz RR, Uyeda A, Gaffney MB, Sheehan KM. Injury prevention education using pictorial information. Pediatrics. [Internet]. 2000; 105 (1): e16-e16. [acesso em: 02 ago. 2016]. Disponível em:

http://pediatrics.aappublications.org/content/105/1/e16.short.

33. Sangvai S, Cipriani L, Colborn DK, Wald ER. Studying injury prevention: practices, problems, and pitfalls in implementation. Clinical pediatrics. [Internet]. 2007; 46 (3): 228-235. [acesso em: 10 ago. 2016]. Disponível em:

http://cpj.sagepub.com/content/46/3/228.full.pdf+html.

34. Bovis SE, Harden T, HotzG.Pilot Study: A Pediatric Pedestrian Safety Curriculum for Preschool Children. J Trauma Nurs. [Internet]. 2016; 23 (5): 247-56. [acesso em: 10 mar. 2017]. Disponível em: https://www.ncbi.nlm.nih.gov/pubmed/27618373. 35. Lehna Carlee et al. Nursing students practice primary fire prevention. Burns. [Internet]. 2013; 39 (6): 1277-1284. [acesso em: 02 ago. 2016]. Disponível em: http://www.sciencedirect.com/science/article/pii/S030541791300034X.

36. Minchom PE, Sibert JR, Newcombe RG, Bowley MA. Does health education prevent childhood accidents? Postgraduate medical journal. [Internet]. 1984; 60 (702): 260-262. [acesso em: 02 ago. 2016]. Disponível em:

http://pmj.bmj.com/content/60/702/260.full.pdf+html.

37. Araújo AR, Gubert FA, Tomé MABG, Martins MC, Fontenele NL, Barros EC. Prevenção de acidentes em uma creche: experiência com pais, professores e pré-escolares. Revista de Enfermagem UFPE OnLine. [Internet]. 2017; 11 (Supl. 4): 1671-8. [acesso em: 10 jun. 2018]. Disponível em: https://periodicos.ufpe.br/revistas/revistaenfermagem/article/view/15264/18063.

38. Souza HVL, Rodrigues RL, Filho IJM, Gomes AS. Discussão sobre as Abordagens Associadas à Aprendizagem Autodirigida e sua Relação com as Tecnologias Educacionais. Revista de Informática Aplicada. [Internet]. 2017; 13 (1): 99-108. [acesso em: 20 abril 2018]. Disponível em: http://www.ria.net.br/index.php/ria/article/view/185/194.

39. Nascimento et al. Acidentes com pré-escolares atendidos em unidade de saúde da família. Revista Eletrônica Gestão \& Saúde. [Internet]. 2015; 6 (02):1174-89. [acesso em: 17 ago. 2018]. Disponível em:

http://periodicos.unb.br/index.php/rgs/article/view/22462/16059.

40. Silva CB, Kantorski KJC, Motta MGC, Pedro ENR. Atividades de educação em saúde junto ao ensino infantil: relato de experiência. Revista de Enfermagem UFPE OnLine. [Internet]. 2017; 111(Supl. 12): 5455-63. [acesso em: 02 jun. 2018]. Disponível em: http://www.lume.ufrgs.br/bitstream/handle/10183/174055/001057033.pdf? sequence=1.

41. Vieira LJES, Carneiro RCMM, Frota MA, Gomes ALA, Ximenes LB. Ações e possibilidades de prevenção de acidentes com crianças em creches de Fortaleza, Ceará. Ciênc. Saúde Coletiva. [Internet]. 2009; 14 (5): 1687-1697. [acesso em: 02 ago. 2016]. Disponível em: http://www.scielo.br/pdf/csc/v14n5/10.pdf. 
42. Margotti E, Costa PPS, Corrêa AMC. A importância da prevenção de acidentes na infância: um relato de experiência. [Internet]. 2018; 7 (1): 200-208. [acesso em: 16 ago. 2018]. Disponível em:

http://seer.uftm.edu.br/revistaeletronica/index.php/enfer/article/view/2281/pdf.

43. Kishimoto TM et al. Jogo, brinquedo, brincadeira e a educação. São Paulo: Cortez Editora, 2017.

44. Piaget J. Sabedoria e ilusões da filosofia. São Paulo: Difusão Européia do Livro; 1967.

45. Vilaronga RS, Sousa OS. A ilustração tátil na contação de história: o programa etnomatemática e o imaginário da criança cega. 90 Encontro Internacional de Formação de Professores e 10ㅇ Fórum Permanente de Inovação Educacional [Internet]. 2016. [acesso em: 23 abril2018]. Disponível em: https://eventos.set.edu.br/index.php/enfope/article/view/2241/657.

46. Dias MP et al. Identificação dos fatores de risco para acidentes na primeira infância no contexto creche. Rev. APS. [Internet]. 2013;16(1): 20-26. [acesso em:16 ago. 2017]. Disponível em: http://aps.ufjf.emnuvens.com.br/aps/article/view/1514/692.

47. Cassemiro JP, Fonseca ABC, Secco FVM. Promover saúde na escola: reflexões a partir de uma revisão sobre saúde escolar na América Latina. Ciência \& Saúde Coletiva. [Internet]. 2014; 19(3): 829-840. [acesso em: 23 abril 2018]. Disponível em: https://www.scielosp.org/pdf/csc/2014.v19n3/829-840/pt.

48. Falkenberg MB, Mendes TPL, Moraes EP, Souza EM. Educação em saúde e educação na saúde: conceitos e implicações para a saúde coletiva. Ciênc. Saúde Coletiva. [Internet] 2014; 19 (3): 847-852. [acesso em: 08 ago. 2016]. Disponível em:

http://www.scielo.br/pdf/csc/v19n3/1413-8123-csc-19-03-00847.pdf.

49. Ferreira, ABH. Dicionário Aurélio da Língua Portuguesa. 5a ed. Curitiba: Positivo, 2014.

50. França EB, et al. Principais causas de mortalidade na infância no Brasil, em 1990 e 2015: estimativas do estudo de Carga Global de Doença. Rev Bras Epidemiol. [Internet]. 2017; 20 (1): 46-60. [acesso em 12 mar. 2017]. Disponível em:

http://www.scielo.br/pdf/rbepid/v20s1/1980-5497-rbepid-20-s1-00046.pdf. 